\title{
Competências colaborativas e o uso de Tecnologias da Informação e Comunicação: PET-Saúde/Interprofissionalidade em período de pandemia
}

\author{
Collaborative competencies and the use of Information and Communication Technologies: pet \\ health interprofissionality in pandemic period \\ Competencias colaborativas y uso de Tecnologías de Información y Comunicación: \\ interprofisionalidad sanitaria de las mascotas en período de pandemia
}

Recebido: 25/12/2020 | Revisado: 26/12/2020 | Aceito: 31/12/2020 | Publicado: 04/01/2021

\author{
Jamile Maria Sampaio Tabosa \\ ORCID: https://orcid.org/0000-0003-1385-4091 \\ Universidade Federal do Ceará, Brasil \\ E-mail: jamilemariasampaio@gmail.com \\ Mihatovit Teixeira Monteiro \\ ORCID: https://orcid.org/0000-0002-7336-4716 \\ Universidade Federal do Ceará, Brasil \\ E-mail: mihatovit06@gmail.com \\ Karina Oliveira de Mesquita \\ ORCID: https://orcid.org/0000-0003-1528-5339 \\ Escola de Saúde Pública Visconde de Saboia, Brasil \\ E-mail: karinamesquita1991@gmail.com \\ Taynã Coelho Simões \\ ORCID: https://orcid.org/0000-0002-4579-0347 \\ Centro de Saúde da Família Hebert de Sousa, Brasil \\ E-mail: taynasimoesc@gmail.com \\ Camilla Araújo Lopes Vieira \\ ORCID: https://orcid.org/0000-0003-1706-3772 \\ Universidade Federal do Ceará, Brasil \\ E-mail: tgd.camilla@gmail.com \\ Jacques Antonio Cavalcante Maciel \\ ORCID: https://orcid.org/0000-0002-2293-8433 \\ Universidade Federal do Ceará, Brasil \\ E-mail: jacques.maciel@ sobral.ufc.br \\ Maria Socorro de Araújo Dias \\ ORCID: https://orcid.org/0000-0002-7813-547X \\ Universidade Estadual Vale do Acaraú, Brasil \\ E-mail: socorroad@gmail.com
}

\begin{abstract}
Resumo
Em decorrência da pandemia pela Covid-19 o PET-Saúde/Interprofissionalidade reorganizou seu processo de trabalho, atuando de forma remota com o uso das Tecnologias da Informação e Comunicação (TIC), cumprindo os critérios de distanciamento social e compromisso com a saúde pública. Para tanto, buscou-se neste artigo, evidenciar as competências colaborativas desenvolvidas com o uso das TIC em período de pandemia pelo PETSaúde/Interprofissionalidade. Trata-se de um relato de experiência, do tipo descritivo e com abordagem qualitativa, desenvolvido no município de Sobral, Ceará, Brasil, no período de abril a outubro de 2020. Por meio de recursos tecnológicos foi possível desenvolver competências pertinentes ao ensino, pesquisa e extensão. Nessa perspectiva, pode-se relatar que as competências colaborativas: liderança colaborativa, comunicação interprofissional, funcionamento da equipe, resolução de conflitos, cuidado centrado na comunidade e clareza de papéis foram incorporadas a esse processo. Assim, as TIC permitiram a continuidade dos processos de trabalho e atividades do PETSaúde/Interprofissionalidade no âmbito do tripé ensino, pesquisa e extensão, de forma a contribuir com o enfrentamento da pandemia e sustentabilidade da integração ensino-serviço-comunidade.

Palavras-chave: Tecnologias da informação e comunicação; Educação interprofissional; Competências colaborativas.
\end{abstract}

\section{Abstract}

As a result of the Covid-19 pandemic, PET-Saúde / Interprofessionality reorganized its work process, acting remotely with the use of Information and Communication Technologies (ICT), fulfilling the criteria of social distance and commitment to public health. Therefore, this article sought to highlight the collaborative skills developed with the use of ICT in a pandemic period by PET-Saúde / Interprofessionality. This is an experience report, of a descriptive type 
and with a qualitative approach, developed in the municipality of Sobral, Ceará, Brazil, from April to October 2020. Through technological resources it was possible to develop skills relevant to teaching, research and extension. From this perspective, it can be reported that collaborative competences: collaborative leadership, interprofessional communication, team functioning, conflict resolution, community-centered care and clarity of roles were incorporated into this process. Thus, ICT allowed the continuity of PET-Saúde / Interprofessional work processes and activities within the scope of the teaching, research and extension tripod, in order to contribute to facing the pandemic and sustainability of teaching-service-community integration.

Keywords: Information and communication technologies; Interprofessional education; Collaborative skills.

\section{Resumen}

A raíz de la pandemia Covid-19, PET Saúde / Interprofessionality reorganizó su proceso de trabajo, actuando de forma remota con el uso de Tecnologías de la Información y la Comunicación (TIC), cumpliendo los criterios de distancia social y compromiso con la salud pública. Por tanto, este artículo buscó destacar las habilidades colaborativas desarrolladas con el uso de las TIC en un período pandémico por PET-Saúde / Interprofesionalidad. Se trata de un relato de experiencia, de tipo descriptivo y con enfoque cualitativo, desarrollado en el municipio de Sobral, Ceará, Brasil, de abril a octubre de 2020. A través de recursos tecnológicos fue posible desarrollar habilidades relevantes para la docencia, la investigación y extensión. Desde esta perspectiva, se puede reportar que en este proceso se incorporaron competencias colaborativas: liderazgo colaborativo, comunicación interprofesional, funcionamiento en equipo, resolución de conflictos, atención centrada en la comunidad y claridad de roles. Así, las TIC permitieron la continuidad de los procesos de trabajo y actividades del PET-Saúde / Interprofesionalidad en el ámbito del trípode de docencia, investigación y extensión, con el fin de contribuir al afrontamiento de la pandemia y la sostenibilidad de la integración docencia-servicio-comunidad.

Palabras clave: Tecnologías de la información y la comunicación; Educación interprofesional; Habilidades colaborativas.

\section{Introduçãa}

Em 2019 um surto de SARS-CoV-2 (Severe acute respiratory syndrome coronavirus 2), causador da infecção pelo novo coronavírus (Covid -19), deixou os órgãos de saúde em estado de alerta. Mais tarde infectando rapidamente várias regiões do mundo, a doença tornou-se pandêmica e impactou de maneira diferente as mais diversas camadas sociais (Freitas, Napimoga \& Donalisio, 2020).

Diante do estado de calamidade pública no âmbito da saúde, desconhecendo ainda a manifestação dos efeitos da doença, todas as esferas governamentais buscaram medidas protetivas com o intuito de impedir a propagação do vírus SARS-Cov-2. Baseando-se em dados epidemiológicos foram adotadas providências de acordo com os indicadores de casos da doença em cada local, variando de normas leves, até mais severas. (Garrido \& Garrido, 2020).

A adoção de medidas de proteção individual é muito importante para o controle da disseminação de uma doença com alta taxa de transmissibilidade. Por tratar-se de um novo vírus, com ausência de uma vacina ou tratamento farmacêutico específico, os métodos não farmacológicos tornaram-se essenciais. Por isso, medidas de distanciamento social, isolamento de casos, fechamento de escolas e comércio, restrição de tráfego, uso de máscaras faciais e reforços de higiene, como o hábito de lavar as mãos, estão entre as estratégias mais adotadas pelos governos para reduzir o contágio e evitar o colapso dos sistemas de saúde. (Peixoto et al., 2020).

Em resposta a Pandemia, os processos de integração ensino-serviço-comunidade também necessitaram de readequações, permitindo a continuidade das ações e auxílio às medidas de enfrentamento à pandemia. Assim, as atividades desenvolvidas por docentes, discentes e trabalhadores passaram a ser viabilizadas, em sua maioria, pelas Tecnologias da Informação e Comunicação (TIC). Estas são utilizadas como ferramentas de auxílio ao processo de educação e como instigadoras para a melhoria da aprendizagem. (Ribeiro et al., 2020)

Dessa forma, o Programa de Educação pelo Trabalho para a Saúde (PET-Saúde/Interprofissionalidade) ancorou-se nas ferramentas de educação à distância para manter sua atuação com intuito de proporcionar uma formação reorientada para as práticas de atenção e construção do conhecimento a partir das necessidades vigentes. A reorganização das ações foi pautada por uma reinvenção das práticas de atuação em seu caráter metodológico e vivencial. 
As TIC cada vez mais e de forma rápidas se fazem presentes no cotidiano e estão inseridas nos processos de educação e trabalho. Com o distanciamento vivenciado na pandemia elas têm propiciado maiores possibilidades de disseminação de informações, espaços de interação e novas metodologias de trabalho. No entanto, Amem e Nunes (2006) salientam a importância do desenvolvimento de competências e habilidades nesse processo de apropriação de tais ferramentas.

Em vista do avanço no processo de globalização e nas novas demandas em saúde, é cada vez mais necessário que profissionais e estudantes desenvolvam o conhecimento de modo flexível e colaborativo, que possam desenvolver habilidades e competências para lidar com situações adversas de modo criativo e inovador. De acordo com Barbosa et al. (2020) as transformações nos hábitos e relações de saúde têm requerido que diferentes áreas do conhecimento se integrem e construam uma formação interdisciplinar e um trabalho interprofissional em saúde. Desta maneira, aponta-se para um novo cenário de formação e atuação profissional cunhados pela tecnologia e pela interdisciplinaridade.

Nessa perspectiva, os percalços trilhados pelo PET-Saúde/Interprofissionalidade neste período evidenciam que por meio de recursos tecnológicos foi possível desenvolver competências colaborativas com o uso das TIC. Assim, faz-se pertinente compartilhar tais experiências no intuito de dar continuidade e sustentabilidade aos processos relacionados ao enfrentamento da Covid-19, bem como a outros problemas de saúde pública emergentes.

O objetivo deste artigo é evidenciar as competências colaborativas desenvolvidas com o uso das TIC em período de pandemia pelo PET-Saúde/Interprofissionalidade em um município do Norte Cearense do Brasil.

\section{Metodologia}

Trata-se de um relato de experiência, do tipo descritivo e com abordagem qualitativa vivenciado pelos integrantes do eixo ensino - serviço- comunidade do Programa de Educação pelo Trabalho para a Saúde - PET-Saúde/Interprofissionalidade 2019-2021, representados pelas categorias de Psicologia, Enfermagem, Educação Física, Medicina e Odontologia. A vivência foi desenvolvida no período de abril a outubro de 2020 envolvendo os estudantes, preceptores e tutores do programa do município de Sobral, Ceará, Brasil.

O método científico pode ser compreendido como uma série de regras, um trabalho sistemático, realizado com o intuito de geração de conhecimento para a ciência. O relato de experiência é uma estratégia eficaz para descrição de vivências que os autores buscam apresentar para que contribua com a construção de conhecimento na área de atuação. Em geral, o relato de caso é uma metodologia qualitativa, métodos importantes para a interpretação e reflexão dos pesquisadores. (Pereira et al., 2018)

Foram utilizadas as TIC como ferramentas para viabilizar as ações do Programa, permitindo o desenvolvimento de competências colaborativas no campo do ensino, da pesquisa e da extensão.

No que toca à prática do ensino, foram feitas discussões teórico-conceituais acerca da prática interprofissional e colaborativa, para o aprimoramento do fazer em saúde. Nesse sentido, destaca-se a participação no curso Educação Interprofissional e Prática Colaborativa realizado pela Organização Pan - Americana da Saúde (OPAS), como um recurso que disponibilizou o acesso a materiais atualizados acerca do assunto, versando sobre aspectos teóricos e práticos da interprofissionalidade. Os conteúdos eram discutidos semanalmente pelos grupos de monitores, preceptores e tutores do PETSaúde/Interprofissionalidade, sendo possível a elaboração coletiva de produtos e o planejamento de ações colaborativas.

Reunidos em grupos de estudo pela plataforma Google Meet era feita a explanação e discussão dos conteúdos, de modo a refletir e construir novas percepções. Os principais assuntos abordados incluíram a prática colaborativa, educação interprofissional, suas dimensões contextual, institucional, cultural e relacional e a inserção da interprofissionalidade na formação e no trabalho em saúde. Alguns produtos elaborados pelo grupo foram a construção de uma mandala, mapas mentais, plano de ação e um podcast. Todos estes elaborados por meio de plataformas e recursos tecnológicos que possibilitaram a interação de todos e um fazer colaborativo. 
Na dimensão da pesquisa, destaca-se o processo de construção de um livro intitulado "Educação Interprofissional e Práticas Colaborativas em Saúde" inteiramente digital sobre interprofissionalidade e a prática colaborativa, que apresenta aspectos teórico-metodológicos e vivenciais de todos os grupos tutoriais do PET-Saúde/Interprofissionalidade de Sobral (Dias et al., 2020).

A elaboração do livro envolveu toda a equipe, desde o processo de planejamento até a publicização das experiências. 1) O planejamento inicial se deu pela coordenação geral e tutores, com a organização das ações, seleção de experiências a serem escritas e publicizadas e mediação dos processos de gestão; 2) Em seguida, houve a construção dos capítulos por cada grupo tutorial, configurando em ações coletivas envolvendo todos os atores do PET-Saúde/Interprofissionalidade. Foram realizadas oficinas para escrita dos textos por meio de plataformas como o google meet, google drive, mendeley e plataformas de base de dados para fontes de pesquisa; 3) Por último, houve o processo de revisão dos capítulos, diagramação do livro e publicização pela equipe gestora do PET-Saúde/Interprofissionalidade.

No que se refere à extensão, pode-se relatar os processos criativos idealizados por todos os grupos, para a explanação dos conceitos de Interprofissionalidade e Prática Colaborativa em saúde para a comunidade em geral. Com essa perspectiva, foi criada uma conta na plataforma Instagram, rede social escolhida pelo seu amplo número de usuários e nível de alcance. Para tal, foram elaboradas postagens com ilustrações e ideias de forma criativa e dinâmica, buscando a atenção de diferentes internautas. Além disso, a ferramenta também foi utilizada para a divulgação de eventos onlines voltados para a área de atuação do PETSaúde/Interprofissionalidade, como a participação no evento $14^{\circ}$ Congresso Internacional da Rede Unida cujo a experiência do PET-Saúde/Interprofissionalidade de Sobral representou a região Nordeste na mesa intitulada "Conexões e provocações sobre o agir do trabalho do Pet-Saúde no tecido social” e participação na V Mostra Integração Ensino-Serviço do Sistema Saúde Escola onde apresentou-se a experiência "Uso de tecnologias da informação e comunicação em período de pandemia pelo PetSaúde/Interprofissionalidade". A rede social também serviu como um dispositivo informativo sobre a Covid-19 e seus impactos e ainda para a publicação de campanhas de conscientização, como Setembro Amarelo e Outubro Rosa.

Ainda no âmbito da extensão, foi elaborado um curta-metragem que contou com a participação de monitores, preceptores, tutores e coordenadora do projeto. A produção tinha como propósito os mesmos objetivos das demais publicações, facilitar o acesso à informação de Interprofissionalidade e Prática Colaborativa de maneira dinâmica. Para isso, foi formulado um roteiro e estabelecida uma ordem de participação no vídeo, onde foi abordado inicialmente a prática colaborativa e, em seguida, a interprofissionalidade de acordo com a visão e experiência de cada um. Utilizando uma linguagem fácil e acessível, os participantes fizeram vídeos individuais e, posteriormente, as gravações foram enviadas para um editor, também membro do projeto, que finalizou a produção para que fosse publicada na rede social.

As competências interprofissionais constituem uma abordagem integrativa de descrição das competências necessárias a uma prática de colaboração interprofissional. Assim, podem ser citados 6 domínios de competências que envolvem o conhecimento, habilidades, atitudes e valores que moldam os julgamentos essenciais para uma prática colaborativa interprofissional. Sendo eles: Comunicação interprofissional, cuidado centrado na comunidade, clareza de funções, funcionamento da equipe, liderança colaborativa e resolução de conflitos. Deste modo, para a análise e discussão das informações utilizou-se como referencial teórico as competências colaborativas do Canadian Interprofessional Health Collaborative (CICH), contemplando os impactos das experiências e as competências desenvolvidas nos eixos ensino, pesquisa e extensão.

\section{Resultados e Discussão}

Para fins didáticos, as competências desenvolvidas serão abordadas de acordo com as práticas de ensino, pesquisa e extensão listadas abaixo: 


\subsection{Competências colaborativas desenvolvidas no âmbito do ensino}

Diante do distanciamento social, essencial para o enfrentamento da pandemia, o uso das TIC mostrou-se uma ferramenta necessária para a manutenção dos trabalhos e continuidade de atividades, bem como revigoraram o desenvolvimento de competências da interprofissionalidade (Nelson et al., 2020).

No âmbito do ensino, evidenciou-se o desenvolvimento das seguintes competências colaborativas: Liderança Colaborativa, Comunicação Interprofissional, Funcionamento da equipe e Resolução de Conflitos.

Nesse contexto, durante o progresso do curso, foram trabalhadas algumas competências, entre elas a liderança. Inicialmente as aulas eram ministradas aos tutores de cada grupo e esses eram responsáveis por dar prosseguimento ao ensino, mediando discussões on-line e as tomadas de decisões compartilhadas dos demais membros do grupo, dessa forma, garantindo um trabalho colaborativo. A comunicação interprofissional foi demonstrada durante os debates, sendo uma qualificação importante do grupo para o desenvolvimento de relações confiáveis para o compartilhamento de responsabilidades, através da escuta, reflexão e discussão a partir das opiniões que surgem. A utilização das TIC, nesse aspecto, contribuiu para a colaboração interprofissional e disseminação de informações por todos os segmentos do PET-Saúde/Interprofissionalidade - Tutores, preceptores e monitores, com eficiência e praticidade. Nesse sentido, há uma tendência na utilização de ferramentas digitais de informação que auxiliem no processo de aprendizagem, não apenas no momento da pandemia, mas na vida contemporânea (Coelho, Morais \& Rosa, 2020).

Para a elaboração das atividades, os participantes entenderam o funcionamento da equipe como uma competência essencial para a confecção de produtos. O estabelecimento de relações de trabalho seguras e eficazes, viabilizou a colaboração mútua e respeitosa, sendo acolhidas e discutidas as sugestões realizadas por cada indivíduo com o intuito de contribuir para a proposta das atividades do curso. A resolução de conflitos desenvolveu um nível de consenso entre aqueles com pontos de vista diferentes, permitindo que todos os membros participassem do debate proposto pelas aulas e refletissem as opiniões distintas, buscando as melhores alternativas para a equipe.

Nesse aspecto, Carneiro, Rodrigues, França e Prata (2020) defendem que o ensino mediado pela tecnologia torna-se uma importante estrutura no âmbito do aprendizado, modelo que facilita a participação dos indivíduos, valoriza o trabalho em equipe e viabiliza a realização de atividades de forma colaborativa. Dessa forma, é sugerido que instituições educacionais fortaleçam seus planos de atividades com o prosseguimento dessas modalidades, implementando as TIC no ensino.

\subsection{Competências colaborativas desenvolvidas no âmbito da pesquisa}

No campo da pesquisa, foi possível trabalhar as seguintes competências: Funcionamento da equipe, Liderança Colaborativa e Comunicação Interprofissional.

As competências se desenvolvem de modo interdependente e podem ser implementadas ao longo da vida acadêmica e profissional em saúde de modo que façam parte das práticas de formação e atuação. No tocante ao eixo da pesquisa, como supracitado trabalhou-se a confecção de um livro digital escrito pelos membros do programa.

Segundo Monroe-Wise et al. (2019) as TICs têm transformado a maneira como a pesquisa e os cuidados em saúde são conduzidos e os dados gerenciados, de maneira que as tecnologias tornaram-se componentes da pesquisa e da prestação de cuidados em saúde. Assim, favorecendo o desenvolvimento de competências uma vez que muitas dessas ferramentas de TIC além de permitirem a interação, estão acessíveis e disponíveis gratuitamente na internet ou como software de código aberto.

Todo o processo de construção ocorreu de modo interprofissional, onde a divisão dos capítulos pode contribuir ao desenvolvimento do funcionamento da equipe. Desta maneira, os grupos de trabalho atuaram de modo a entender os princípios que regem a dinâmica de um trabalho de equipe e processos grupais, facilitando a colaboração interprofissional entre os membros (CICH, 2010). Apesar das mudanças na dinâmica dos processos grupais em decorrência do distanciamento social, as equipes 
buscaram trabalhar de modo saudável, e facilitar as discussões e interações entre equipe. Assim, denota-se a importância dos grupos de discussão on-line e partilha do material escrito nos capítulos respeitando a participação de todos, a disponibilidade de recursos digitais, a comunicação aberta, a escuta atenta e principalmente o compromisso com a ética interprofissional.

A liderança a ser desenvolvida em uma prática interprofissional se dá por meio do compartilhamento de decisões e implica que cada membro seja responsável por suas ações, responsabilidades e funções (CICH, 2010). Desta forma, por meio de plataformas virtuais como o google drive, a escrita ocorria de modo compartilhado e todos da equipe poderiam acompanhar o seu desenvolvimento, sendo necessário a responsabilidade de cada participante para atingir os resultados, garantir o avanço das relações de trabalho interdependente e o estabelecimento de um clima de prática colaborativa.

Uma outra competência trabalhada foi a comunicação interprofissional. Por meio das TIC buscou-se dar continuidade de modo virtual a rede de comunicação entre os membros, utilizando-se das metas compartilhadas de trabalho de pesquisa, formulação de dados e escrita e a colaboração entre participantes do processo. Tal habilidade é essencial nesse ínterim visto que a comunicação se dá com membros de profissões diferentes, pacientes, famílias e comunidades de forma ágil e responsável. (Lima et al., 2020). Em uma pesquisa acerca de um curso de educação interprofissional e prática colaborativa de modo on-line Jones, Vidal e Taylor (2020) apresentam alguns benefícios desta modalidade como o trabalho em seu próprio ritmo, conexões interpessoais, aprendizagem aberta, envolvimento com o conteúdo e tempo para reflexão. Dentre as dificuldades listou-se a interação face a face, dificuldades técnicas, falta de conexão e envolvimento presencial com colegas e profissionais e os desafios de comunicação.

\subsection{Competências colaborativas desenvolvidas no âmbito da extensão}

No campo da extensão, trabalhou-se as seguintes competências: Cuidado Centrado na Comunidade e Clareza de Papéis.

No que se refere à extensão, as TIC foram essenciais para o alcance da comunidade e desenvolvimento de atividades e projetos que buscam democratizar conhecimentos e informações. $O$ trabalho colaborativo em equipe possibilitou a elaboração de materiais para a publicação na rede social Instagram, entre eles a produção de um curta-metragem.

Desse modo, o cuidado centrado na comunidade foi uma importante competência fortalecida no decorrer das ações. Compartilhar informações e levar educação em saúde para população em geral de forma compreensiva estimula a participação dos usuários no planejamento em saúde, uma vez que o povo é especialista em suas próprias necessidades, favorecendo assim a criação de um plano que atenda às demandas da comunidade, através do diálogo e com a maior participação da população (Coelho, Morais \& Rosa, 2020).

Para a produção do curta-metragem foram utilizados diferentes programas para edição do vídeo, que foi produzido a partir de gravações realizados por representantes de cada categoria: coordenador geral, tutor, preceptores e monitores, onde cada um relatou sua experiência com a prática colaborativa e interprofissionalidade a partir da sua condição. Nesse aspecto, a clareza dos papéis pode ser evidenciada através da capacidade de articulação on-line entre as diferentes categorias, onde os conhecimentos e habilidades específicas construídas a partir da identidade de cada área puderam ser respeitadas e acolhidas no contexto do trabalho colaborativo. Essas atividades remotas também foram viabilizadas através de tecnologias como os smartphones, que facilitaram a gravação e participação dos membros na produção audiovisual. A utilização desses recursos digitais já era algo conhecido pela sociedade, mas se intensificou no período pandêmico, colocando-se como uma alternativa a ser implementada no período que sucederia o isolamento social (Moreira et al., 2020).

Desse modo, percebe-se como as competências são independentes e se desenvolvem simultaneamente, fortalecendo a prática colaborativa e a interprofissionalidade entre os membros do grupo tutorial e aqueles atingidos pelas ações. Nesse aspecto, as TIC têm se mostrado ferramentas eficazes para suprir as necessidades de continuidade de atividades e planejamento de ações, apesar dos desafios que apresenta, como a constante inovação, algumas vezes não é acompanhada pelos usuários, e o acesso 
limitado a algumas camadas sociais. (Fonsêca, 2018)

\section{Considerações Finais}

Em decorrência da pandemia e das medidas de enfrentamento a Covid-19, o PET-Saúde/Interprofissionalidade buscou atuar de forma remota cumprindo os critérios de distanciamento social e compromisso com a saúde pública. Assim, as TIC puderam auxiliar para que os processos relacionados ao ensino e trabalho em saúde tivessem continuidade e possibilitaram aos envolvidos o desenvolvimento de competências colaborativas.

Essas competências por sua vez, são interdependentes e se desenvolvem simultaneamente, fortalecendo a prática colaborativa e a interprofissionalidade entre os membros do grupo tutorial e àqueles atingidos pelas ações. Nesse sentido, as TIC têm se mostrado ferramentas eficazes para suprir as necessidades de continuidade de atividades e planejamento de ações, permitindo a interação e o diálogo de modo virtual.

Perante a nova realidade, muitos desafios se fizeram presentes, dentre eles a habituação a muitas ferramentas virtuais e quais utilizar, a interação face a face e dificuldades de comunicação, de acesso e conexão. Todavia, esse período destacou-se também pelas novas modalidades de interação e aprendizado de formas outras de trabalho e ensino no campo da saúde. Nessa perspectiva, pode-se relatar que as competências colaborativas: liderança colaborativa, a comunicação interprofissional, funcionamento da equipe, resolução de conflitos, cuidado centrado na comunidade e a clareza de papéis foram incorporadas a esse processo.

Diante da experiência relatada e dos ganhos advindos do uso das TIC para o desenvolvimento de competências colaborativas acredita-se viável dar continuidade a esse processo e garantir a sustentabilidade e continuidade dos projetos de ensino e trabalho do programa PET-Saúde/Interprofissionalidade bem como de outros programas e órgãos que vierem a utilizar ferramentas virtuais, nesse contexto e em um período de pós pandemia.

No tocante a experiência, ressalta-se a necessidade de estudos vindouros que abordem o uso de tecnologias no campo interprofissional e prática colaborativa e uma possível sistematização das plataformas mais viáveis aos trabalhos de equipe e uso de competências colaborativas. Além disso, diante do crescente desenvolvimento das TIC e das novas formas de interação, de ensino e trabalho, serão necessários trabalhos que discutam essa perspectiva em um período pós pandemia elencando os desafios, prejuízos e ganhos.

\section{Referências}

Amem, B. M. V., \& Nunes, L. C. (2006). Tecnologias de informação e comunicação: contribuições para o processo interdisciplinar no ensino superior. Revista Brasileira de Educação Médica, 30(3), 171-180. https://www.scielo.br/scielo.php?pid=S0100-55022006000300008\&script=sci_arttext\&tlng=pt.

Barbosa, L.; Jr, Gama, I. C. S., de Oliveira, B. F., Pessalacia, J. D. R., Martins, T. C. R., \& dos Santos, E. M. (2020). Uso de tecnologias na formação interprofissional de acadêmicos de Medicina durante a pandemia de COVID-19. Research, Society and Development, 9(11), e2179119594-e2179119594. https://rsdjournal.org/index.php/rsd/article/view/9594.

Canadian Interprofessional Health Collaborative. (2010). A national interprofessional competency framework. The Collaborative.

Carneiro, L. de A., Rodrigues, W., França, G., \& Prata, D. N. (2020). Use of technologies in Brazilian public higher education in times of pandemic COVID19. Research, Society and Development, 9(8), e267985485. Uso de tecnologias no ensino superior público brasileiro em tempos de pandemia COVID-19. https://rsdjournal.org/index.php/rsd/article/view/5485.

Coelho, A. L., de Araujo Morais, I., \& Silva Rosa, W. V. (2020). A utilização de tecnologias da informação em saúde para o enfrentamento da pandemia do Covid-19 no Brasil. Cadernos Ibero-Americanos de https://www.cadernos.prodisa.fiocruz.br/index.php/cadernos/article/view/709.

Dias, M. S. A., Vieira, C. A. L; Freitas, C. A. S. L., Farias, M. R. de., Lira, R. C. M., Lomeo, R. da C., \& Saraiva, M. J. G. (2020). Educação Interprofissional e Práticas Colaborativas em Saúde. Sobral: Edições UVA.

Fonsêca, R. M. da. (2020). Educação interprofissional em saúde e o desenvolvimento de competências colaborativas na formação em enfermagem e medicina. Dissertação de Mestrado Profissional em Ensino na Saúde, Centro de Ciências da Saúde, Universidade Federal do Rio Grande do Norte, Natal. https://repositorio.ufrn.br/jspui/handle/123456789/25871. 
Freitas, A. R. R., Napimoga, M., \& Donalisio, M. R. (2020). Análise da gravidade da pandemia de Covid-19. Epidemiologia e Serviços de Saúde, 29 , e2020119. https://www.scielosp.org/article/ress/2020.v29n2/e2020119/.

Garrido, R. G., \& Garrido, F. D. S. R. G. (2020). COVID-19: Um panorama com ênfase em medidas restritivas de contato interpessoal. Interfaces CientíficasSaúde e Ambiente, 8(2), 127-141. https://periodicos.set.edu.br/saude/article/view/8640/0.

Jones, T. A., Vidal, G., \& Taylor, C. (2020). Interprofessional education during the COVID-19 pandemic: finding the good in a bad situation. Journal of interprofessional care, 34(5), 633-646. https://www.tandfonline.com/doi/full/10.1080/13561820.2020.1801614.

Lima, A. W. S. D., Alves, F. A. P., Linhares, F. M. P., Costa, M. V. D., Coriolano-Marinus, M. W. D. L., \& Lima, L. S. D. (2020). Percepção e manifestação de competências colaborativas em discentes da graduação em saúde. Revista Latino-Americana de Enfermagem, 28. https://www.scielo.br/scielo.php?pid=S0104-11692020000100305\&script=sci_arttext\&tlng=pt.

Monroe-Wise, A., Kinuthia, J., Fuller, S., Dunbar, M., Masuda, D., Opiyo, E., \& Osoti, A. (2019). Improving Information and Communications Technology (ICT) Knowledge and Skills to Develop Health Research Capacity in Kenya. Online Journal of Public Health Informatics, 11(3). https://www.ncbi.nlm.nih.gov/pmc/articles/PMC6975540/.

Moreira, M. E. S., Silva Cruz, I. L., Sales, M. E. N., Moreira, N. I. T., de Castro Freire, H., Martins, G. A., \& Popolim, R. S. (2020). Metodologias e tecnologias para educação em tempos de pandemia COVID-19/Methodologies and technologies for education in times of pandemic COVID-19. Brazilian Journal of Health Review, 3(3), 6281-6290. https://www.brazilianjournals.com/index.php/BJHR/article/view/11584.

Nelson, I. C. A. de S. R., Bezerra, K. P., Costa, K. F. de L., Oliveira, L. C. de., Vieira, A. N., Fernandes, A. C. L., Queiroz, J. C. de., \& Lima, M. F. do A. P. (2020). Tecnologias de informação e comunicação na atenção à saúde mental de profissionais da saúde no contexto da pandemia da COVID-19 Research, Society and Development, 9(10), e1249108192. https://rsdjournal.org/index.php/rsd/article/view/8192.

Peixoto, S. V., Nascimento-Souza, M. A., Mambrini, J. V. D. M., Andrade, F. B. D., Malta, D. C., \& Lima-Costa, M. F. (2020). Comportamentos em saúde e adoção de medidas de proteção individual durante a pandemia do novo coronavírus: iniciativa ELSI-COVID-19. Cadernos de Saúde Pública, 36, e00195420. https://www.scielosp.org/article/csp/2020.v36suppl3/e00195420/.

Pereira, A. S., Shitsuka, D. M., Parreira, F. J., \& Shitsuka R. (2018). Metodologia da pesquisa científica. [e-book]. Santa Maria. Ed. UAB/NTE/UFSM. https://repositorio.ufsm.br/bitstream/handle/1/15824/Lic_Computacao_Metodologia-Pesquisa-Cientifica.pdf?sequence=1.

Ribeiro, A. M. F., Scorsin, G. R. B., Ravaze, M. A. D. O. F., Donato, R. S., Plentz, L. P., Martins, T. C. R., \& Pessalacia, J. D. R. (2020). Aplicação da metodologia da problematização com arco de Maguerez na formação interprofissional por meio de tecnologias de informação. Saúde e meio ambiente: revista interdisciplinar, 9(Supl. 1), 25-26. http://www.periodicos.unc.br/index.php/sma/article/view/3398. 